\title{
Notch2 controls hepatocyte-derived cholangiocarcinoma formation in mice
}

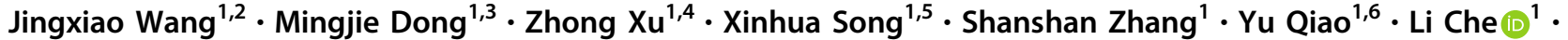 \\ John Gordan ${ }^{1} \cdot$ Kaiwen $\mathrm{Hu}^{2} \cdot$ Yan $\mathrm{Liu}^{3} \cdot$ Diego F. Calvisi ${ }^{7} \cdot$ Xin Chen $\mathbb{1}^{1}$
}

Received: 21 September 2017 / Revised: 01 December 2017 / Accepted: 06 February 2018 / Published online: 16 March 2018

(c) The Author(s) 2018. This article is published with open access

\begin{abstract}
Liver cancer comprises a group of malignant tumors, among which hepatocellular carcinoma (HCC) and intrahepatic cholangiocarcinoma (ICC) are the most common. ICC is especially pernicious and associated with poor clinical outcome. Studies have shown that a subset of human ICCs may originate from mature hepatocytes. However, the mechanisms driving the trans-differentiation of hepatocytes into malignant cholangiocytes remain poorly defined. We adopted lineage tracing techniques and an established murine hepatocyte-derived ICC model by hydrodynamic injection of activated forms of AKT

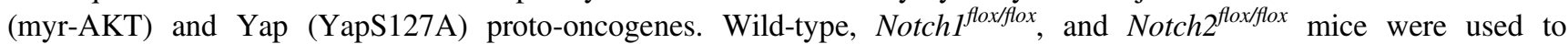
investigate the role of canonical Notch signaling and Notch receptors in AKT/Yap-driven ICC formation. Human ICC and HCC cell lines were transfected with siRNA against Notch2 to determine whether Notch2 regulates biliary marker expression in liver tumor cells. We found that AKT/Yap-induced ICC formation is hepatocyte derived and this process is strictly dependent on the canonical Notch signaling pathway in vivo. Deletion of Notch2 in AKT/Yap-induced tumors switched the phenotype from ICC to hepatocellular adenoma-like lesions, while inactivation of Notchl in hepatocytes did not result in significant histomorphological changes. Finally, in vitro studies revealed that Notch2 silencing in ICC and HCC cell lines down-regulates the expression of Sox 9 and EpCAM biliary markers. Notch2 is the major determinant of hepatocyte-derived ICC formation in mice.
\end{abstract}

These authors contributed equally: Jingxiao Wang and Mingjie Dong

Electronic supplementary material The online version of this article (https://doi.org/10.1038/s41388-018-0188-1) contains supplementary material, which is available to authorized users.

Yan Liu

13911798288@163.com

$\triangle$ Diego F. Calvisi

diego.calvisi@uni-greifswald.de

$\triangle$ Xin Chen

xin.chen@ucsf.edu

1 Department of Bioengineering and Therapeutic Sciences, University of California, San Francisco, San Francisco, USA

2 Dongfang Hospital, Beijing University of Chinese Medicine, Beijing, China

\section{Introduction}

Primary liver cancer is the second most common cause of cancer mortality in the world, with increasing incidence globally [1, 2]. Hepatocellular carcinoma (HCC) and intrahepatic cholangiocarcinoma (ICC) are the two most prevalent liver tumor types. Most ICCs are diagnosed at advanced stage and only a few patients are suitable for surgery at the time of diagnosis. For patients with

3307 Hospital of Academy of Military Medical Science, Beijing, China

4 Department of Gastroenterology, Guizhou Provincial People's Hospital, Guizhou, China

5 Beijing Advanced Innovation Center for Food Nutrition and Human Health, College of Food Science and Nutritional Engineering, China Agricultural University, Beijing, China

6 Department of Oncology, Beijing Hospital National Center of Gerontology Beijing, China

7 Institut für Pathologie, Universitätsmedizin Greifswald, Greifswald, Germany 


\section{A R26R-EYFP mice}

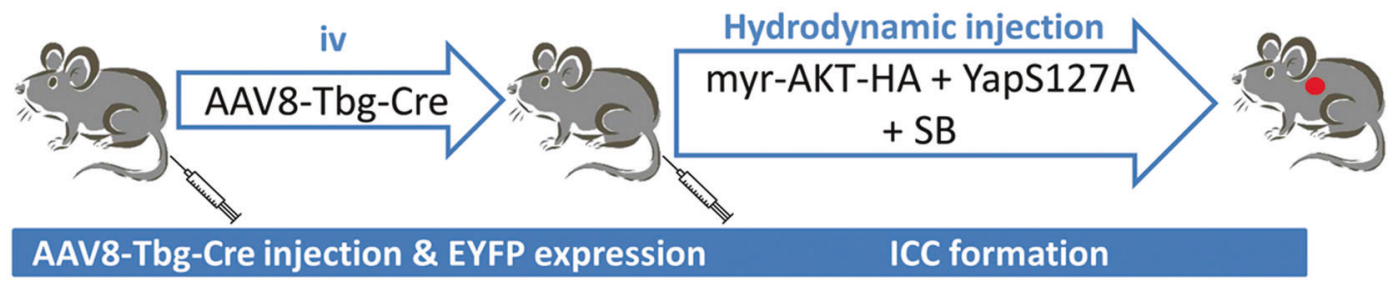

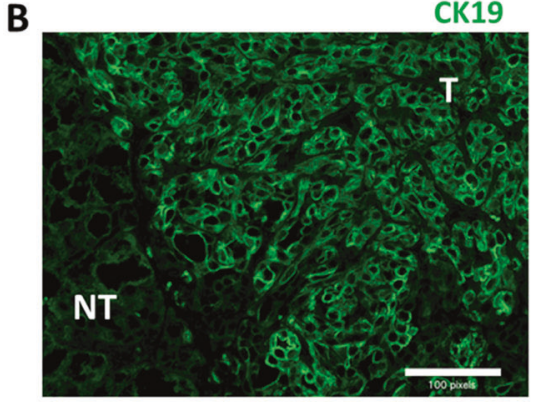

HA tag

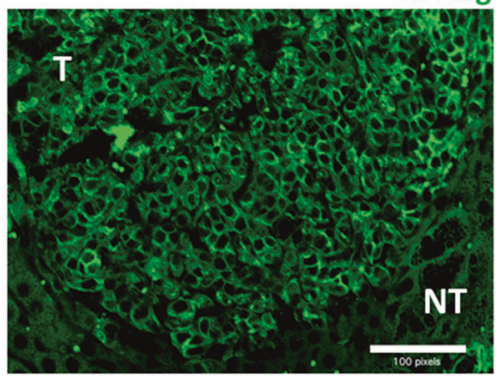

NT

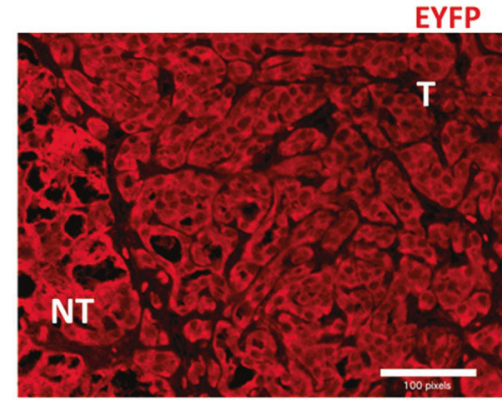

EYFP

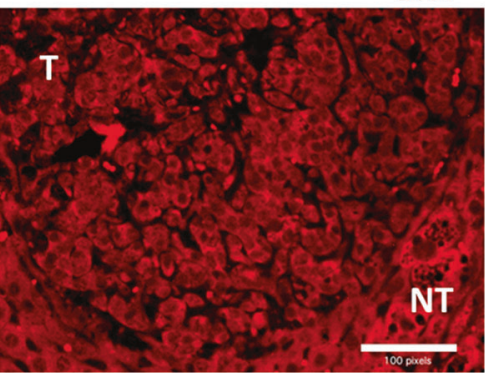

CK19/EYFP/DAPI

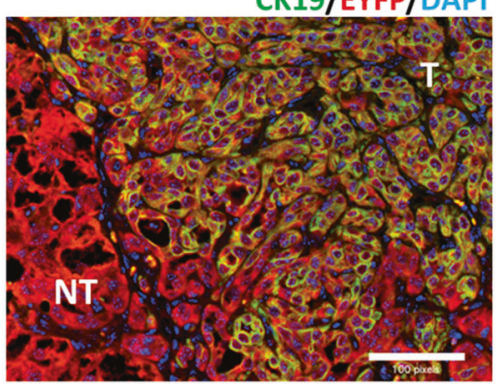

HA tag/EYFP/DAPI

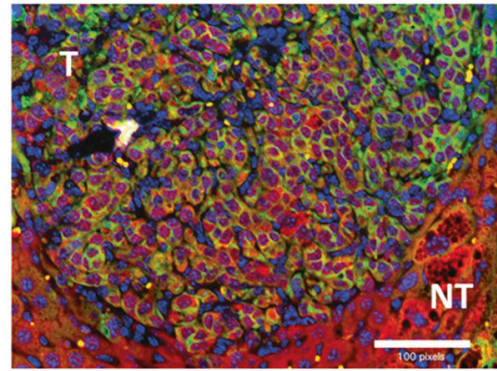

Fig. 1 AKT/Yap-induced intrahepatic cholangiocarcinomas (ICC) derived from hepatocytes. a Study design. AAV-Tbg-Cre was injected intravenously to generate EYFP expression. AKT and Yap were coinjected hydrodynamically to induce tumor development. b The upper panels show immunofluorescence of CK19 and EYFP. The lower

inoperable ICC, very limited treatment options exist. According to the American Cancer Society (www.cancer. org), the 5-year survival rate for ICC patients with localized disease is $\sim 15 \%$, and only $2 \%$ for patients with distal metastasis. ICC has been traditionally considered to be derived from biliary epithelial cells (BEC). However, recent studies have indicated that adult hepatocytes can transdifferentiate into BEC-like cell in various chronic liver diseases, which then may further develop into malignant cells $[3,4]$. In accordance with this hypothesis, recent epidemiology studies have shown that in Western countries, where biliary tract infection rate is extraordinarily low, chronic infections by hepatitis $\mathrm{B}$ or $\mathrm{C}$ virus as well as alcohol abuse are major risk factors for ICC [5], as described for HCC. Studies from our and other laboratories also confirmed that ICC can originate from mature hepatocytes in mice following activation of the Notch signaling $[6,7]$.

Notch is a highly conserved pathway during development. This pathway is critical for biliary cell coordination panels are representative staining of HA tag and EYFP. Note that EYFP was only expressed in hepatocytes, while surrounding mesenchymal cells did not show red fluorescence. Tumor is labeled as $\mathrm{T}$ and non-tumor is labeled as NT. Magnification: 100x

and tubule formation [8]. The structure, homeostasis, and carcinogenesis of the liver relies on the Notch cascade [9, 10]. In mammals, canonical Notch pathway consists of four receptors (Notch1, Notch2, Notch3, and Notch4) and mainly two types of ligands, Serrate/Jagged (Jagged1 and Jagged2) and Delta-like (DLL1, DLL3, and DLL4) [11-13]. This cascade is activated by direct cell-cell interaction, with subsequent cleavage of the Notch receptor extracellular domain (NECD). This structural change leads to the release of the Notch intracellular domain (NICD), which translocates into the nucleus and recruits coactivators, such as Mastermind-like proteins (MAML1, MAML2, or MAML3). Together with the recombinant signal-binding protein for immunoglobulin kappa $\mathrm{J}$ region (RBPJ) transcription factor, they form the transcription complex responsible for the induction of Notch target genes [9, 14]. The most studied Notch signaling targets are hairy/enhancer of split (Hes) and hairy/enhancer of split related with YRPW motif (Hey) families. Another emerging target is 

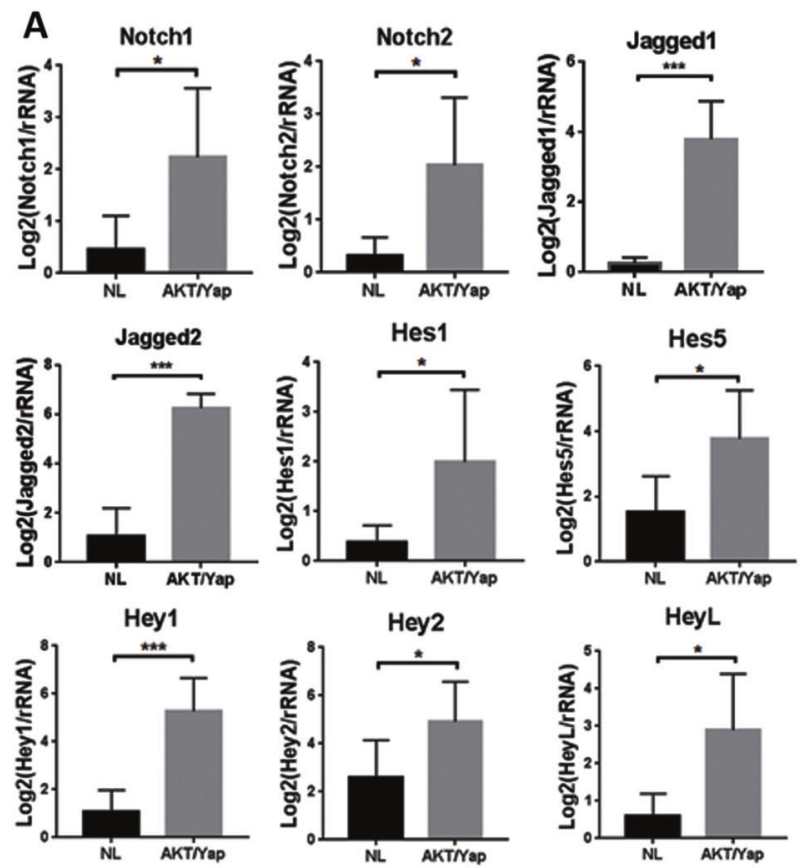

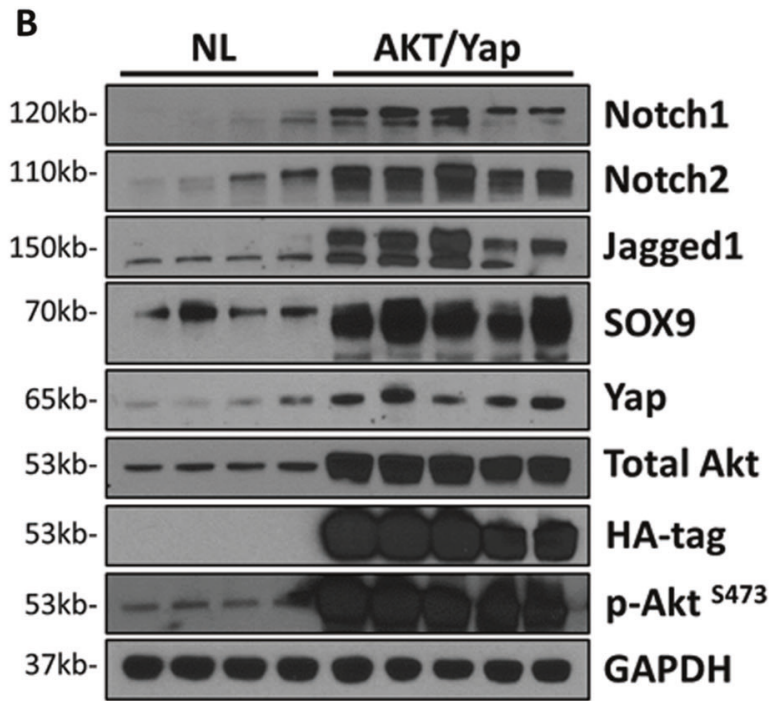

C
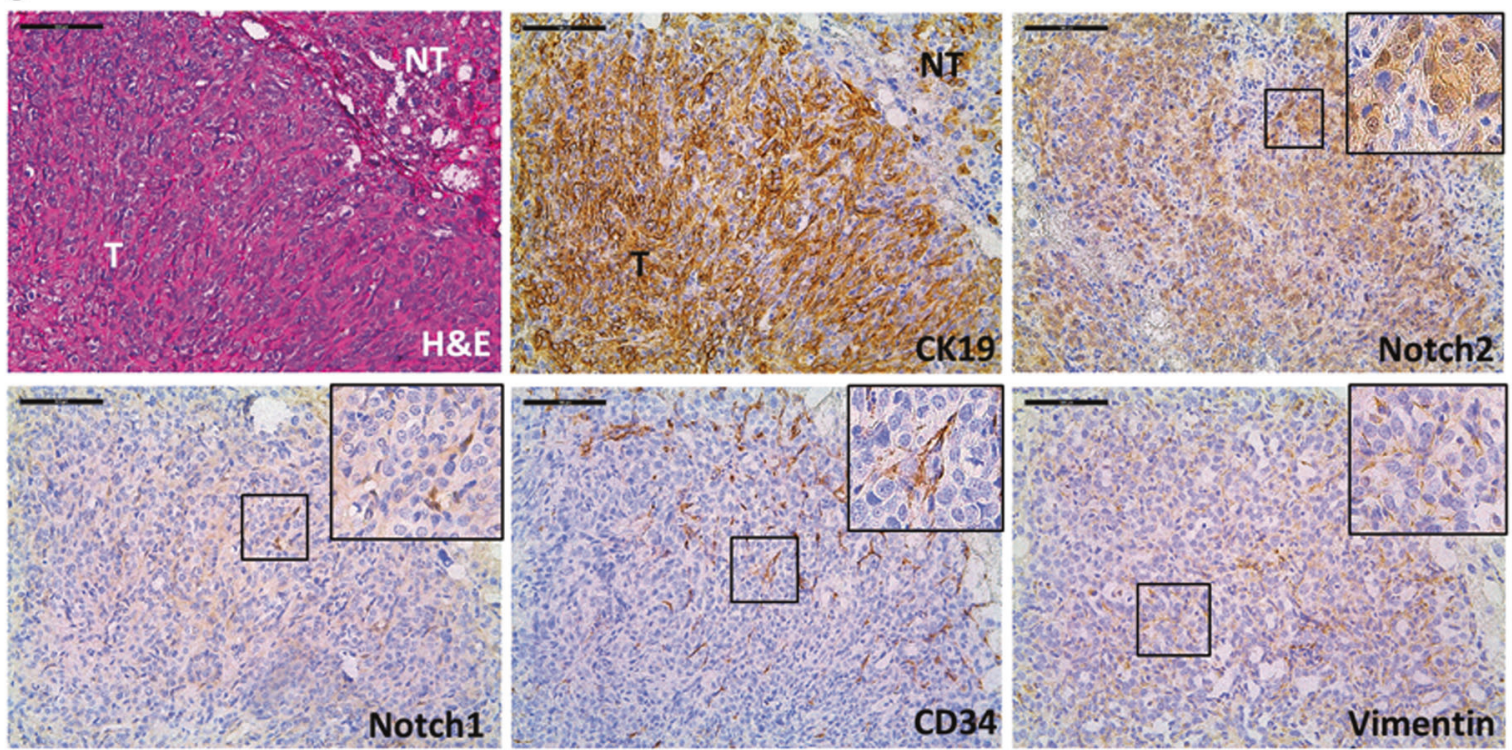

Fig. 2 Notch signaling activation in intrahepatic cholangiocarcinoma (ICC) from AKT/Yap mice. a Notch signaling activation at transcriptional level is presented by qPCR. Data are analyzed and normalized using the $-\Delta \Delta \mathrm{Ct}$ method and presented as mean $\pm \mathrm{SD}$; NL represents normal liver; $n=6, * p<0.05 ; * * p<0.01 ; * * * p<0.001$. b Western blot analysis of relative protein expression in normal liver and tumor samples. GAPDH was used as a loading control. c Hematoxylin

Notch-regulated ankyrin repeat protein (Nrarp), which is activated by the RBPJ-dependent Notch pathway [15].

Both Notch1 and Notch2 receptors are expressed in the liver, but whether they play distinct or redundant roles along hepatocarcinogenesis remains an unanswered issue. Notch1 is considered a tumor suppressor in HCC [16, 17], but a and eosin (H\&E) staining and immunohistochemistry (IHC) of AKT/ Yap-induced tumors. CK19 and Ki67 showed the ICC nature and the proliferative features of these tumors, respectively. Notch2 immunolabeling was localized in the cytoplasm and nucleus of ICC cells. Notch1 positive cells exhibited the same staining pattern of CD34 positive cells, which are mostly endothelial cells. Magnification: 200x; Scale bar: $100 \mu \mathrm{m}$

bona fide oncogene in ICC [18]. Deprivation of Notch1 results in continuous proliferation of hepatocytes [19]. Notch2, on the other hand, seems to be crucial for the differentiation of BECs and is required for normal perinatal and postnatal intrahepatic bile duct (IHBD) development $[20,21]$. The canonical Notch2 signaling can determine 
A

$\mathrm{FVB} / \mathrm{N}$ mice

Sacrifice when large

tumors develop

Control Group
$(n=5)$
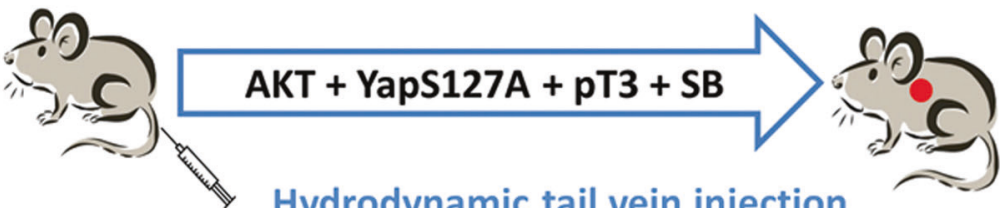

Hydrodynamic tail vein injection

Group $(n=5)$

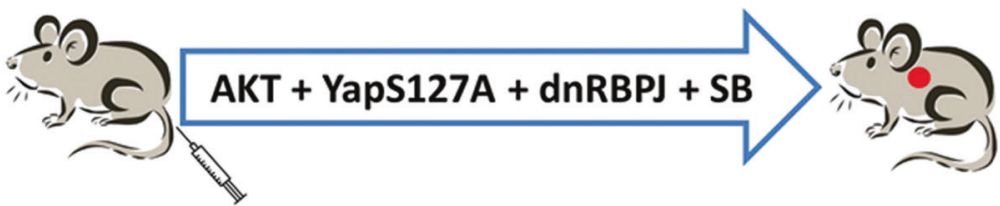

B AKT/Yap/pT3

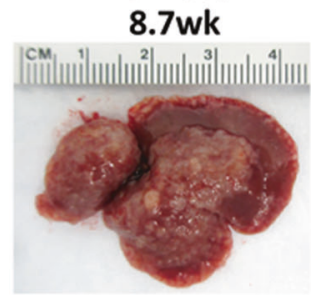

AKT/Yap/dnRBPJ

$10 w k$

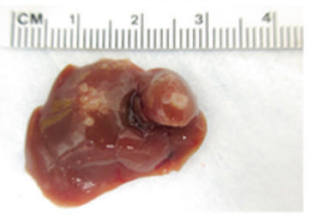

C

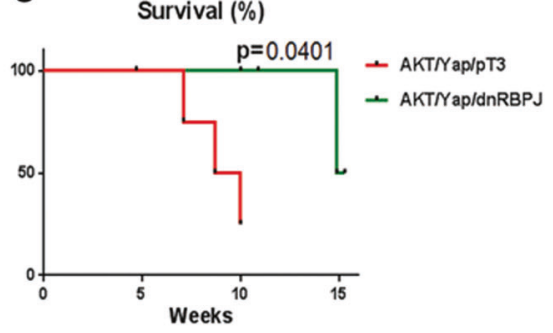

D

AKT/Yap/pT3
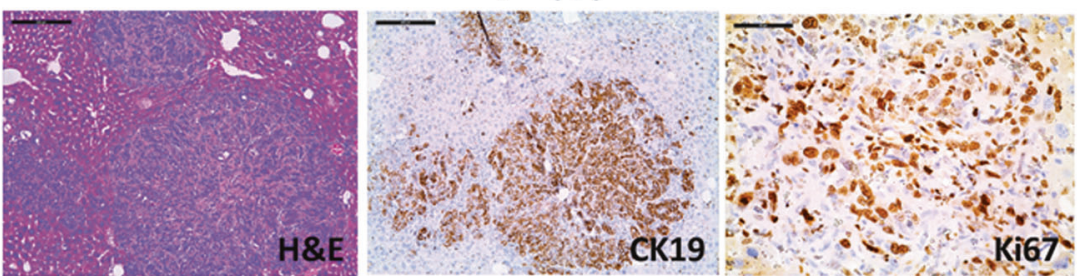

E

F \% Area of $\mathrm{CK} 19+$
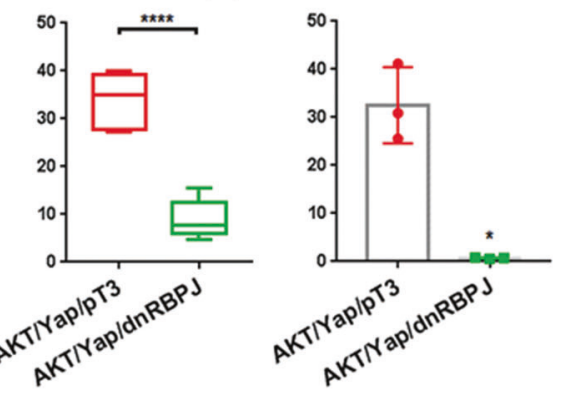

AKT/Yap/dnRBPJ
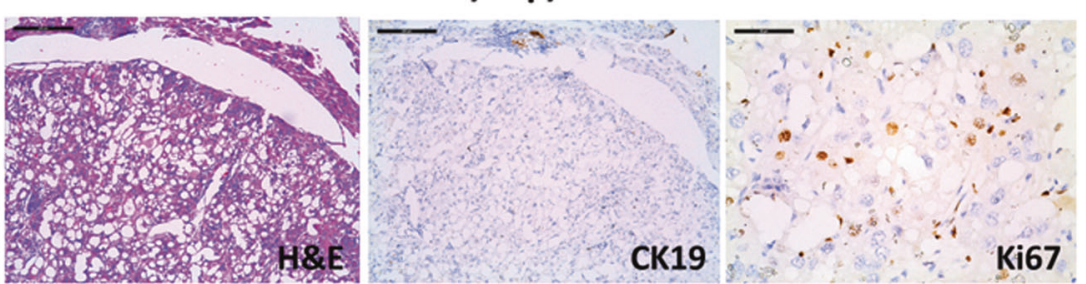
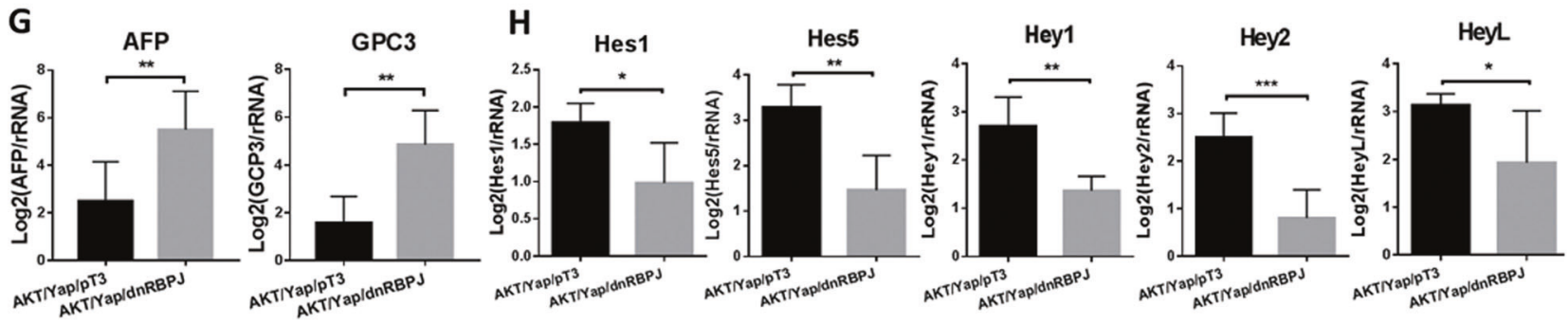

Fig. 3 Intrahepatic cholangiocarcinoma (ICC) formation depends on canonical Notch pathway in AKT/Yap mice. a Study design. b Gross image of livers from each group. c Survival analysis of mice bearing AKT/Yap/pT3 $(n=5)$ and AKT/Yap/dnRBPJ $(n=5)$ tumors as assessed by the Kaplan-Meier survival method. d Upper panels show $\mathrm{H} \& \mathrm{E}$ and immunohistochemistry of AKT/Yap/pT3 mice, and lower panels that of AKT/Yap/dnRBPJ mice, respectively. Scale bar: $100 \mu \mathrm{m}$ for $200 \times$; $50 \mu \mathrm{m}$ for $400 \times$. e The percentage of Ki67 positive cells in the two groups ( $n=5$ per each cohort) was analyzed using Image Pro Plus. f Percentage of CK19 positive area in the two groups is displayed. Area fraction was analyzed with the ImageJ software; $n=3$ per each group. $\mathbf{g}$ Relative mRNA expression of AFP and GPC3 was analyzed and normalized using the $-\Delta \Delta \mathrm{Ct}$ method. $\mathbf{h}$ Relative expression of Notch target genes is shown. All data are presented as mean \pm SD. $* p<0.05 ; * * p<0.01 ; * * * p<0.001 ; * * * * p<0.0001$ 
biliary cell fate of not only embryonic hepatoblasts, but also mature hepatocytes [22]. Furthermore, the expression of Notch2 is more often observed in well-differentiated ICC, indicating its role in biliary tumor cell differentiation [23]. In a recent study [24], we found that anti-Notch2 treatment can reduce both HCC and ICC tumor load induced by AKT and neuroblastoma RAS viral oncogene homolog (NRas) oncoproteins, whereas Notch1 suppression decrease HCC and augments ICC occurrence. This body of evidence suggests the conflicting functions of Notch1 and Notch2 receptors in different contexts.

Formerly, most of the studies regarding Notch1 and Notch2 receptors aimed at their overexpression rather than their suppression. In the present study, we sought to better define the cell functions of Notch1 and Notch2 receptors in mice by loss-of-function experiments. Thus, we generated a mouse model characterized by biliary trans-differentiation of hepatocytes, and investigated whether this cellular event depends on autocrine Notch1 or Notch2 signaling. Specifically, we overexpressed activated forms of v-akt murine thymoma viral oncogene homolog (myr-AKT) and yesassociated protein (YapS127A) genes in hepatocytes by hydrodynamic injection (AKT/Yap) [25]. By adopting lineage tracing technology [6], we show that AKT/Yapinduced ICC formation is hepatocyte derived and this process depends on the canonical Notch signaling pathway. Furthermore, we found that Notch2 is the major driver of the biliary phenotype in AKT/Yap tumors, whereas inactivation of Notch1 slightly delays tumor development without affecting the histological features of AKT/Yap ICC lesions.

\section{Results}

\section{AKT/Yap-induced ICCs originate from hepatocytes}

To determine whether ICC cells induced by AKT/Yap coexpression originate from mature hepatocytes, we applied the hepatocyte fate tracing model as previously described $[6,26]$. Specifically, we injected adeno-associated virus encoding Cre-recombinase under the control of hepatocytespecific thyroxine-binding globulin (Tbg) promoter (AAV8Tbg-Cre) into mice that carry EYFP disrupted by a floxed stop codon in the ubiquitously expressed Rosa26 locus (R26R-EYFP mice). This procedure leads to the activation of hepatocyte-specific EYFP expression after 1 week of AAV injection. We applied hydrodynamic tail vein injection of hemagglutinin (HA) tagged AKT and YapS127 plasmid (AKT/Yap), along with Sleeping Beauty (SB) plasmids to initiate ICC development (Fig. 1a). Immunofluorescence (IF) staining showed that all tumor cells were positive for the BEC marker CK19, hepatocyte lineage marker EYFP, as well as ectopically expressed HA tagged AKT (Fig. 1b). The results demonstrate that AKT/Yap coexpression induces hepatocyte-derived ICCs in the mouse liver.

\section{Notch signaling cascade is activated in AKT/Yap ICC}

As the canonical Notch signaling has been implicated in cholangiocarcinogenesis [14, 27], especially hepatocytederived ICC formation [6, 7], we evaluated whether the Notch cascade is activated in AKT/Yap ICC tumors. We found that AKT/Yap ICCs express higher mRNA levels of Notch receptors (Notch1 and Notch2), Notch ligands (Jagged1 and Jagged2), and canonical Notch target genes (Hes1, Hes5, Hey1, Hey2, and HeyL) when compared to normal liver (Fig. 2a). Western blot analysis confirmed the upregulation of Notch1, Notch2, Jag1 as well as the BEC marker Sox9 in AKT/Yap tumors (Fig. 2b). To further characterize the specific cell type(s) within the tumors that express Notch receptors, Notch1 and Notch2 immunohistochemistry (IHC) was performed. We observed Notch2 immunoreactivity in the cytoplasm and nucleus of tumor cells, with no Notch2 expression being detected in mesenchymal cells. In contrast, Notch1 immunolabeling could be easily observed in cells surrounding the tumor nodules, similar to that of CD34, which marks endothelial cells (ECs) (Fig. 2c). Upon longer DAB (3,3'-diaminobenzidine) incubation time, we could detect weak cytoplasmic and/or membranous staining of Notch1 in tumor cells, as well as in surrounding non-neoplastic hepatocytes (Sup Fig. 1).

Altogether, our data underline the activation of the Notch cascade in AKT/Yap ICC. Specifically, Notch2 is strongly expressed in ICC cells, whereas Notch1 is predominantly expressed in the cells of the tumor microenvironment.

\section{AKT/Yap-induced ICC depends on the canonical Notch pathway}

Next, we tested whether the canonical Notch pathway is a major pathogenic player in AKT/Yap-driven cholangiocarcinogenesis. For this purpose, we co-expressed AKT and Yap plasmids with a dominant negative form of recombination signal-binding protein for immunoglobulin kappa $\mathbf{J}$ (dnRBPJ) in FVB/N mice, which will be referred to as AKT/Yap/dnRBPJ (Fig. 3a). RBPJ is a downstream transcription factor that constitutes the DNA-binding site of the Notch transcription complex. Expression of dnRBPJ has been shown to effectively block the canonical Notch signaling $[28,29]$. Additional mice were injected with AKT and Yap with pT3-EF1 $\alpha$ empty vector as control (AKT/ Yap/pT3) (Fig. 3a). Of note, we found that blocking the canonical Notch cascade significantly delayed AKT/Yap- 

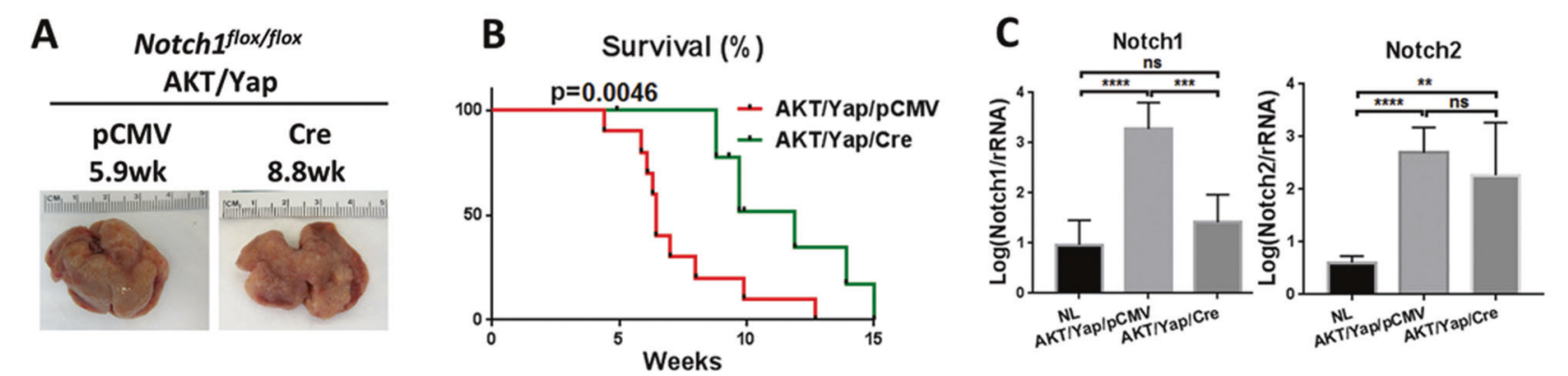

E
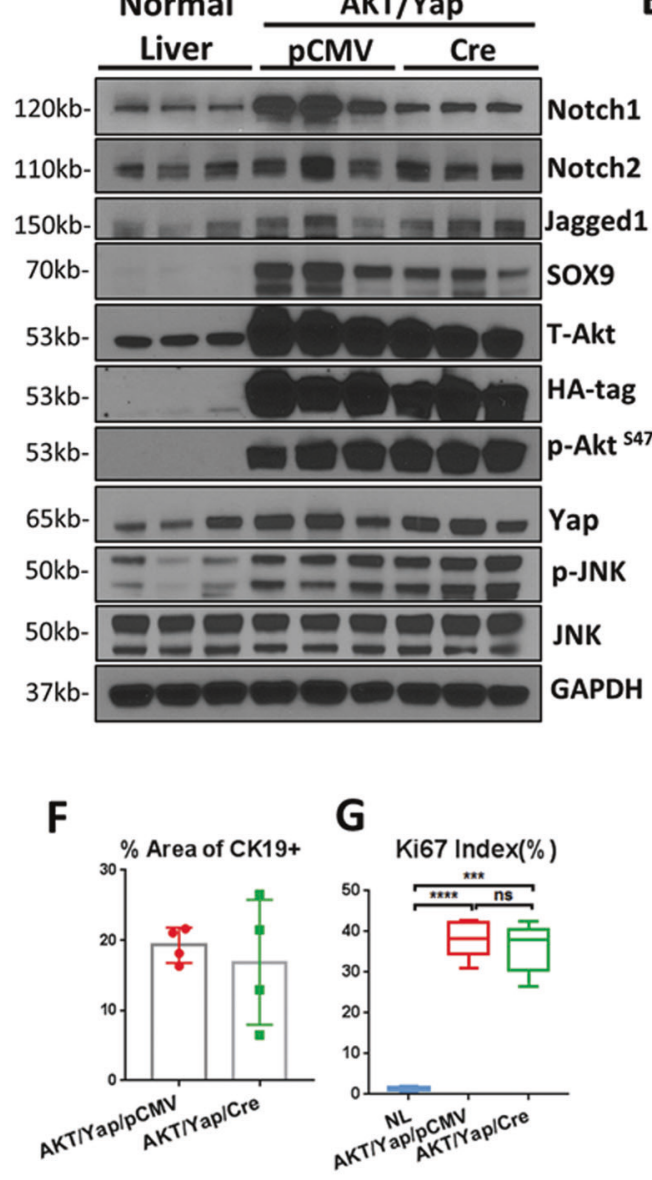

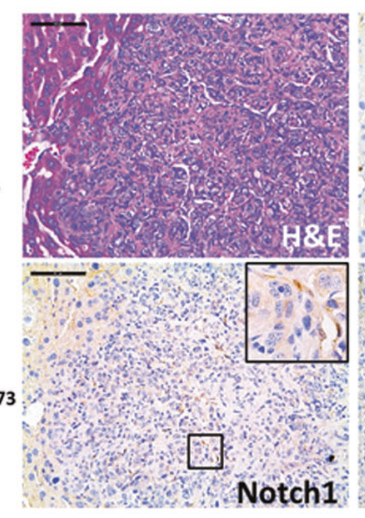

AKT/YapS127A/pCMV

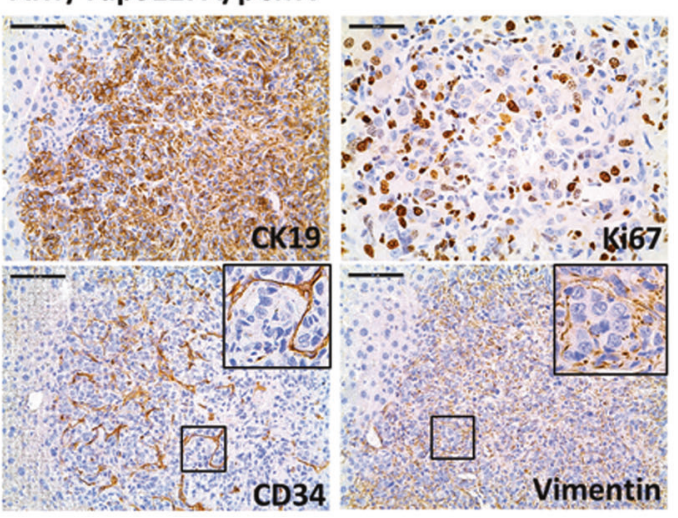

АKT/YapS127A/Cre
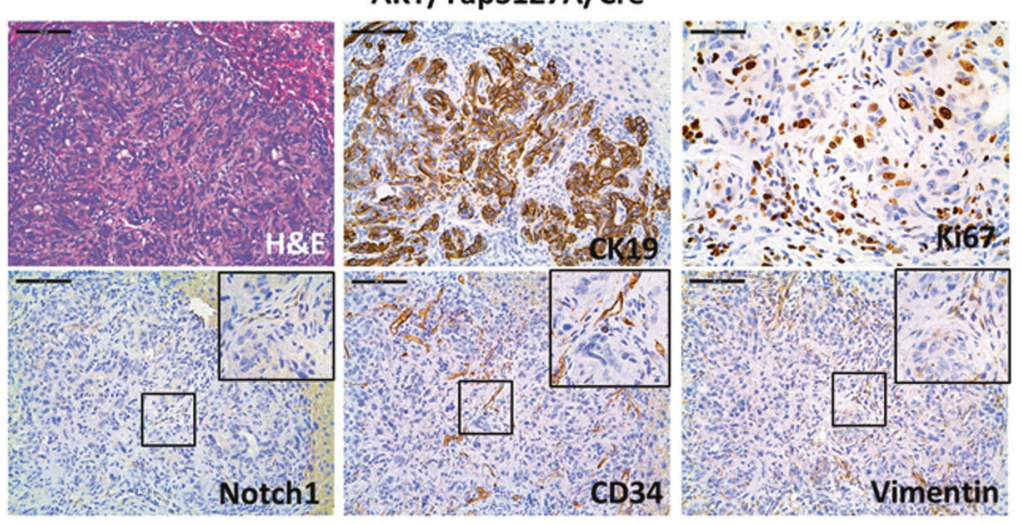

H
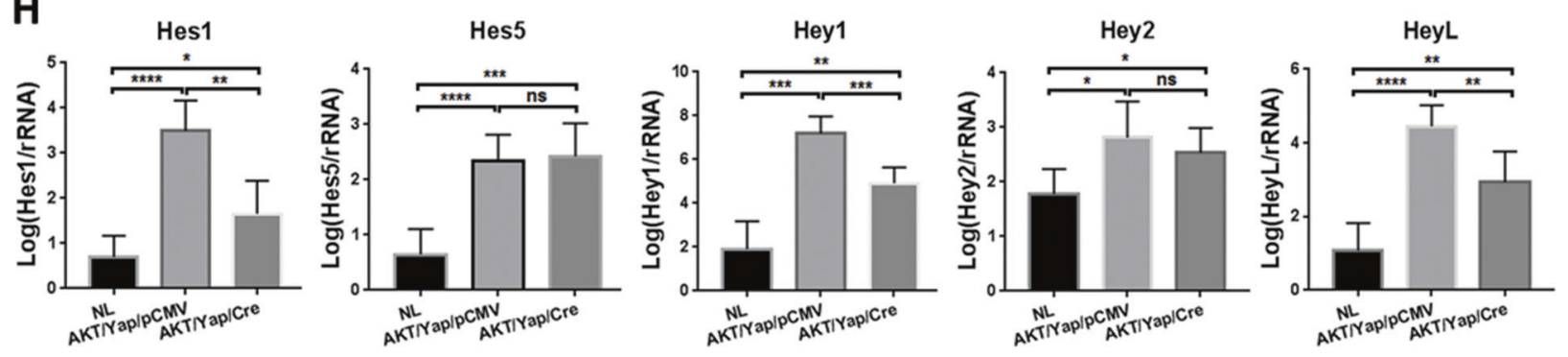

induced liver tumor formation and prolonged mouse survival (Sup Fig. 2 and Fig. 3b,c). Consistently, Ki67 index significantly decreased in AKT/Yap/dnRBPJ liver tumors when compared to that in AKT/Yap/pT3 liver tumors (Fig. 3d,e), implying that tumor cell proliferation was downregulated by blocking the canonical Notch signaling. Most 
Fig. 4 Notch1 inactivation does not prevent ICC formation in AKT/ Yap mice. a Gross images of livers from AKT/Yap/pCMV and AKT/ Yap/Cre mice. b Survival analysis of Notch $1^{\text {floxflox }}$ mice bearing AKT/ Yap/pCMV $(n=10)$ and AKT/Yap/Cre $(n=10)$ tumors using the Kaplan-Meier survival method. c Relative mRNA expression of Notch1 and Notch2 was analyzed and normalized using the $-\Delta \Delta \mathrm{Ct}$ method and presented as mean $\pm \mathrm{SD}$; NL represents normal liver; $n=$ 6. d Western blot analysis of normal liver, AKT/Yap/pCMV, and AKT/Yap/Cre mice. GAPDH was used as a loading control. e H\&E and immunohistochemical staining of AKT/Yap/pCMV (upper panels) and AKT/Yap/Cre (lower panels) mice. Several sections were enlarged for a better view on the expression of Notch1, CD34, and Vimentin. Scale bar: $100 \mu \mathrm{m}$ for $200 \times ; 50 \mu \mathrm{m}$ for $400 \times$. f Quantification of CK19 + area percentage is displayed, $n=4$. g Percentage of Ki67 positive nuclei was analyzed in normal liver $(n=5), \mathrm{AKT} / \mathrm{Yap} / \mathrm{pCMV}(n=5)$, and AKT/Yap/Cre $(n=5)$ mice. $\mathbf{h}$ Relative expression of Notch target genes is shown; $n=6$. All data are presented as mean \pm SD. ns $p>$ $0.05 ; * p<0.05 ; * * p<0.01 ; * * * p<0.001 ; * * * * p<0.0001$

importantly, histological analysis revealed that while AKT/ Yap/pT3 tumors consisted of pure ICC, as also indicated by strong immunoreactivity for the CK19 biliary marker (Fig. 3d,f), AKT/Yap/dnRBPJ liver tumors consisted mostly of hepatocellular adenomas and few HCC lesions. These observations were further underscored by increased levels of tumor hepatocellular markers, such as alpha-fetoprotein (AFP) and glypican 3 (GPC3), in AKT/Yap/dnRBPJ tumors (Fig. 3g). As expected, the downstream targets of the Notch signaling were down-regulated in AKT/Yap/dnRBPJ mice (Fig. 3h), thus substantiating the effective blockade of this cascade by dnRBPJ overexpression.

Taken together, our study demonstrates that the canonical Notch signaling is required for AKT/Yap-induced hepatocyte-derived ICC formation in mice.

\section{Limited effects of cell autonomous Notch1 signaling in AKT/Yap-induced ICC formation in mice}

Next, we investigated whether Notch1 or Notch2 was the major Notch receptor mediating AKT/Yap-induced ICC development. As our study shows the low expression of Notch1 in normal hepatocytes and ICC tumor cells (Sup Fig. 1), we first investigated the cell autonomous role of Notch1. Thus, Notch $1^{\text {floxflox }}$ mice were hydrodynamically injected with AKT, YapS127A, and pCMV/Cre plasmids to allow the expression of AKT/Yap oncogenes in Notch1 knockout hepatocytes (AKT/Yap/Cre; Sup Fig. 3). Notch $1^{\text {flox/flox }}$ mice injected with AKT, Yap, and pCMV (empty vector) were used as control (AKT/Yap/pCMV; Sup Fig. 3). Hepatocyte-specific ablation of Notch1 delayed AKT/Yap-induced liver tumor development (Fig. 4a,b). qRT-PCR and Western blot analyses demonstrate that Notch1 mRNA and protein expression were both lower in $\mathrm{AKT} / \mathrm{Yap} / \mathrm{Cre}$ mouse liver tumors than that in control AKT/ Yap/pCMV tumors (Fig. 4c,d). However, substantial Notch1 expression could still be observed in AKT/Yap/Cre tumors, suggesting that most of Notch1 was not expressed in tumor cells, but in other cell types within the ICC lesions. Consistent with this observation, IHC revealed that Notch1 could still be detected in AKT/Yap/Cre mouse liver tumors, and most likely in CD34(+) ECs (Fig. 4e). Histological analysis showed that tumor-specific ablation of Notchl did not change tumor morphology: indeed, ICC lesions were found throughout the liver and no hepatocellular tumors, including hepatocellular adenoma and HCC, were observed in both AKT/Yap/Cre and AKT/Yap/pCMV Notchl flox/flox mice (Fig. 4e). The overall ICC tumor burden, as measured by the percentage of CK19(+) liver area, did not differ in the two cohorts (Fig. 4f). Similarly, no difference in cell proliferation was observed (Fig. 4g). Finally, loss of Notchl in tumor cells did not affect the protein expression of Notch2, Jag1, and BEC marker Sox9 as well as mRNA levels of Notch targets, including Hes5 and Hey2 (Fig. 4c,d, h). Loss of Notch1 instead decreased the expression of Hes1, Hey, and HeyL (Fig. 4h).

In summary, our study demonstrates that Notch1 expression is low in AKT/Yap tumor cells, and ablation of Notchl in hepatocytes delays ICC tumorigenesis. However, the cell autonomous Notch1 signaling is neither a major determinant of activated canonical Notch cascade nor the key driver for AKT/Yap-induced ICC development in mice.

\section{Cell autonomous Notch2 deprivation is required for AKT/Yap-induced ICC development in mice}

In light of the pivotal role of Notch 2 signaling in biliary cell fate determination [22], we investigated the function of Notch2 in AKT/Yap ICC lesions using conditional Notch $2^{\text {flox/flox }}$ mice (Sup Fig. 4). Specifically, we hydrodynamically injected AKT, Yap, and Cre into Notch $2^{\text {flox/flox }}$ mice (AKT/Yap/Cre). Notch2 flox/flox mice injected with AKT, Yap, and pCMV empty vector (AKT/Yap/pCMV) were used as control. Ablation of Notch2 significantly delayed AKT/Yap-induced ICC development in mice, although eventually all mice developed lethal burden of liver tumors and were required to be euthanized (Fig. 5a,b). Histologically, ICC lesions could be found throughout the liver parenchyma of AKT/Yap/pCMV mice, while no hepatocellular lesions were identified. Strikingly, ablation of Notch2 resulted in mostly hepatocellular adenomas with some HCC lesions in the liver, while no ICC lesions could be detected (Fig. 5c). These findings were further validated via IHC of BEC marker CK19 and hepatocyte-specific marker HNF-4 $\alpha$ (Fig. 5c,d). Proliferation of tumor cells was significantly reduced following Notch2 depletion, as indicated by $\mathrm{Ki} 67$ index (Fig. 5e). Western blot analysis confirmed the loss of Notch2 in the liver tumor lesions (Fig. 5f). In addition, mRNA levels of the canonical Notch targets, including Hes1, Hes5, Hey1, Hey2, and HeyL were 


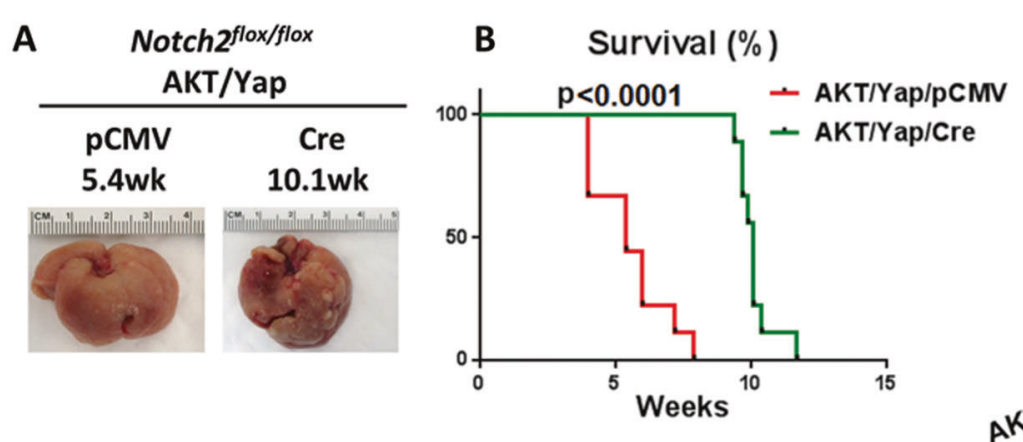

D \% Area of $\mathrm{CK} 19+$ E Ki67 Index(\%)

C
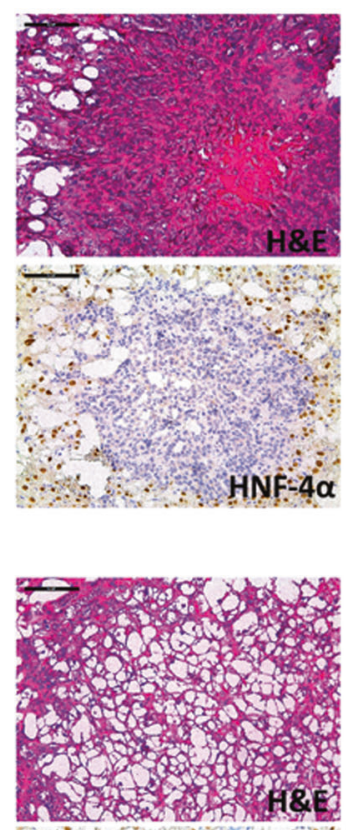

HNF- $4 \alpha$

\section{AKT/Yap/pCMV}

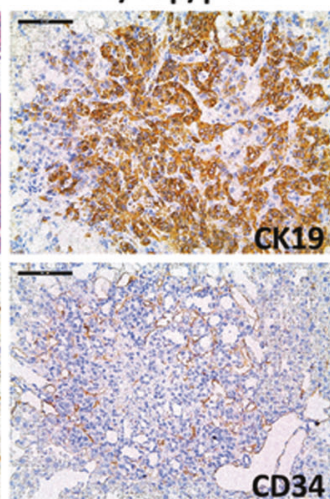

AKT/Yap/Cre

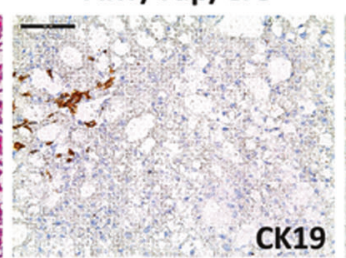

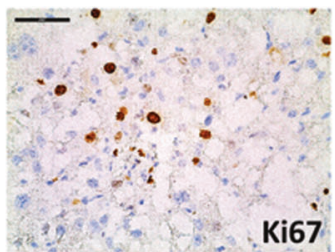

Ki67
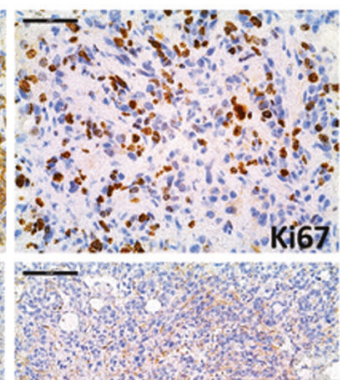

Vimentin

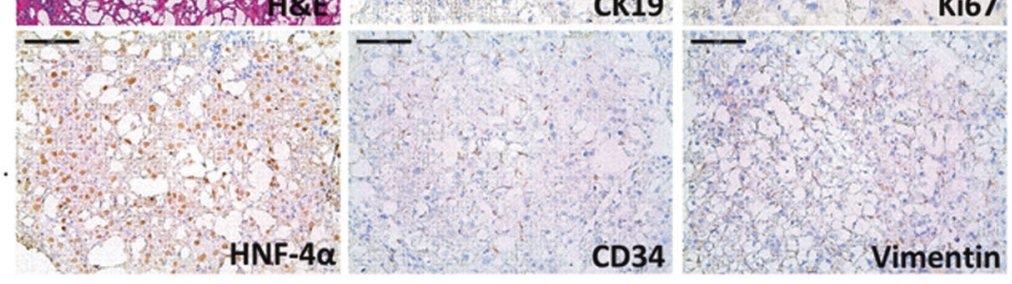

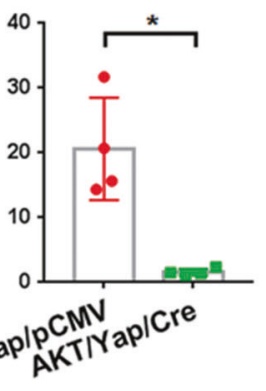

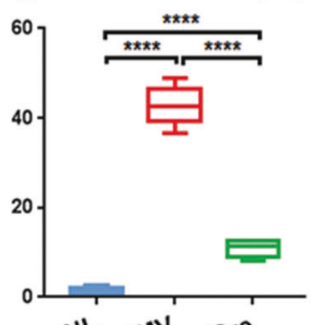

NL
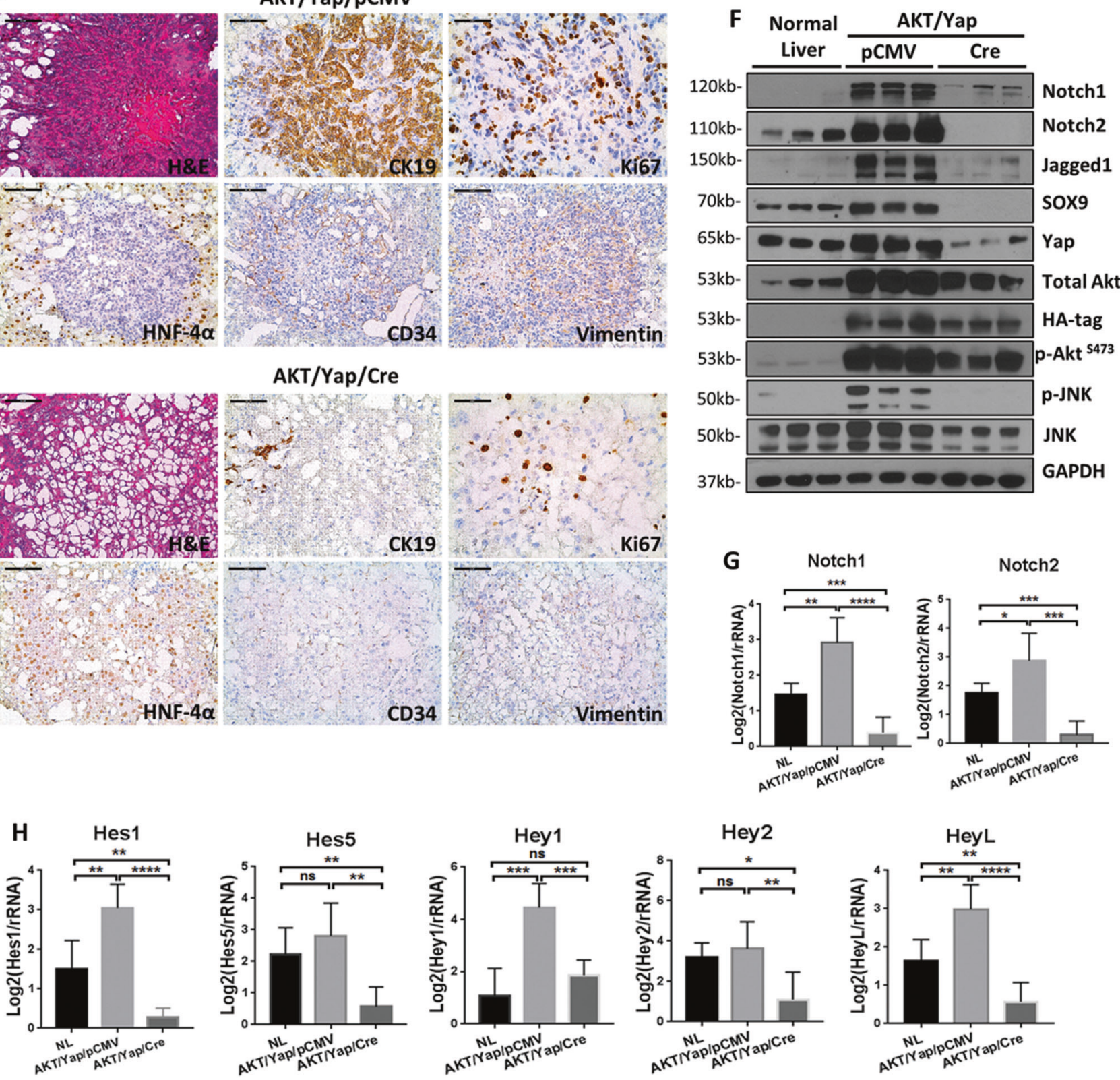
Fig. 5 Notch2 inactivation suppresses ICC development in AKT/Yap mice. a Gross images of AKT/Yap/pCMV and AKT/Yap/Cre livers. b Survival analysis of Notch $2^{\text {flox/flox }}$ mice bearing AKT/Yap/pCMV $(n=$ 9) and AKT/Yap/Cre $(n=9)$ tumors. c H\&E and immunohistochemistry of AKT/Yap/pCMV (upper panels) and AKT/Yap/Cre (lower panels) mice. Several sections were enlarged for optimal vision. Scale bar: $100 \mu \mathrm{m}$ for $200 \times ; 50 \mu \mathrm{m}$ for $400 \times$. d CK19 staining was quantified and represents the percentage of positive staining area of the whole area in the two groups, $n=4$. e Percentage of Ki67 positive cells was analyzed in normal liver $(n=5), \mathrm{AKT} / \mathrm{Yap} / \mathrm{pCMV}(n=6)$, and AKT/ Yap/Cre $(n=6)$ livers. f Western blotting of normal liver, AKT/Yap/ pCMV, and AKT/Yap/Cre livers in Notch2 $2^{\text {floxflox }}$ mice. GAPDH was used as a loading control. g Relative expression of Notch1 and Notch2 using qPCR. Data were analyzed and normalized using the $-\Delta \Delta \mathrm{Ct}$ method, $n=6$. $\mathbf{h}$ Relative expression of Notch signaling target genes using qPCR; $n=6$. All data are presented as mean \pm SD. ns $p>0.05$; ${ }^{*} p<0.05 ; * * p<0.01 ; * * * p<0.001 ; * * * * p<0.0001$

remarkably dowregulated following Notch2 deletion, as assessed by qRT-PCR analysis (Fig. 5g,h). These data indicate that Notch2 is the major regulator of the canonical Notch cascade in this mouse model. Interestingly, Notch1 expression was also decreased upon Notch2 ablation (Fig. $5 \mathrm{f}, \mathrm{g})$. Immunolabeling of CD34 and Vimentin in the tumor sections revealed that ECs and fibroblast cells were less numerous in AKT/Yap/Cre lesions (Fig. 5c). Since Notch1 is most likely expressed dominantly in ECs, the reduced ECs may account for the decreased Notch1 expression in AKT/Yap/Cre lesions.

Recent experimental data point to the JNK signaling as a critical modulator of biliary differentiation and proliferation [30]. Accordingly, we found that the JNK pathway was effectively inactivated following Notch2 (Fig. 5f) but not Notch1 (Fig. 4d) deletion, thus suggesting that the JNK cascade could be a crucial downstream effector of Notch2 in ICC.

Altogether, these data indicate that Notch2 is essential for Akt/Yap-induced ICC development.

\section{Deletion of Notch2 undermines the biliary properties in vitro}

The studies in mice suggest the possibility that Notch2 controls BEC-like cell fate in human liver tumor cells. Thus, we tested whether silencing of Notch2 affected BEC marker expression in human HCC and ICC cell lines. A total of three ICC cell lines (KKU-M213, HuCC-T1, and RBE) and three HCC cell lines (HLE, SNU-449, and SNU475) were transfected with scrambled control siRNA or siNotch2. As expected, Notch2 expression was impaired following siNotch 2 treatment in both ICC and HCC cell lines (Fig. 6a, b). Also, knockdown of Notch2 was paralleled by decreased SOX9 expression as well as by decline of canonical Notch targets, including Hes1, Hey1, and Nrarp (Fig. 6c).
Transcriptional levels of Epcam were significantly reduced in all HCC cell lines and two ICC cell lines.

In summary, our study indicates that Notch2 expression, at least partially, controls BEC fate gene expression in human HCC and ICC tumor cells.

\section{Discussion}

ICC is a malignant form of liver tumor lacking effective treatment options. The cellular origin of ICC is currently a matter of debate. Previously, it was believed that ICC arises from BEC of the liver. This is most likely the case in patients where a predisposing risk factor is limited to the biliary ducts, such as infections by Clonorchis sinensis, choledocholithiasis, or choledochal cysts. However, in Western and East Asian countries, where inflammatory processes affecting the biliary tract are uncommon, HBV and $\mathrm{HCV}$ infection and alcohol abuse are the main risk factors for ICC, as for HCC [5]. Since HBV and HCV viruses do not infect BECs, how ICC develops in the context of chronic hepatocyte injury remains a matter of debate. Presumably, hepatocytes are the cells of origin of this subset of human ICCs. Previous studies have shown the extraordinary plasticity of mature hepatocytes, which can transdifferentiate into BECs upon injury [4, 31, 32]. In the presence of additional oncogenic and/or mutagenic stimuli, it is possible that these trans-differentiated BECs further transform into ICC cells. Besides hepatocytes, several other cell types can also undergo trans-differentiation, leading to cancer formation [33]. One of such examples is pancreatic ductal adenocarcinoma (PDAC) [34, 35]. Like ICC, PDAC was thought to originate from ductal cells within the pancreas. However, recent studies have challenged this assumption, showing that acinar cells (the major parenchymal cells in the pancreas) are able to transdifferentiate into ductal cells. This process, also known as acinar-toductal metaplasia, can facilitate pancreas regeneration after injury $[34,35]$. These metaplastic cells have been found to be precursors of pre-neoplastic PanIN lesions, which can further progress to PDAC [34, 35]. In this study, we show that AKT/Yap-induced ICCs arise from mouse hepatocytes. Our previous investigation has demonstrated the frequent concomitant activation of AKT and Yap signaling cascades in human ICCs [25]. Thus, we believe that the AKT/Yap mouse model is highly relevant to study human cholangiocarcinogenesis, especially in the context of chronic liver injury where hepatocytes may in fact be the target of transformation.

The molecular mechanisms underlying the development of hepatocyte-derived ICC remain poorly understood. Previous studies from our and other groups identified the canonical Notch cascade as a critical driver in hepatocyte- 

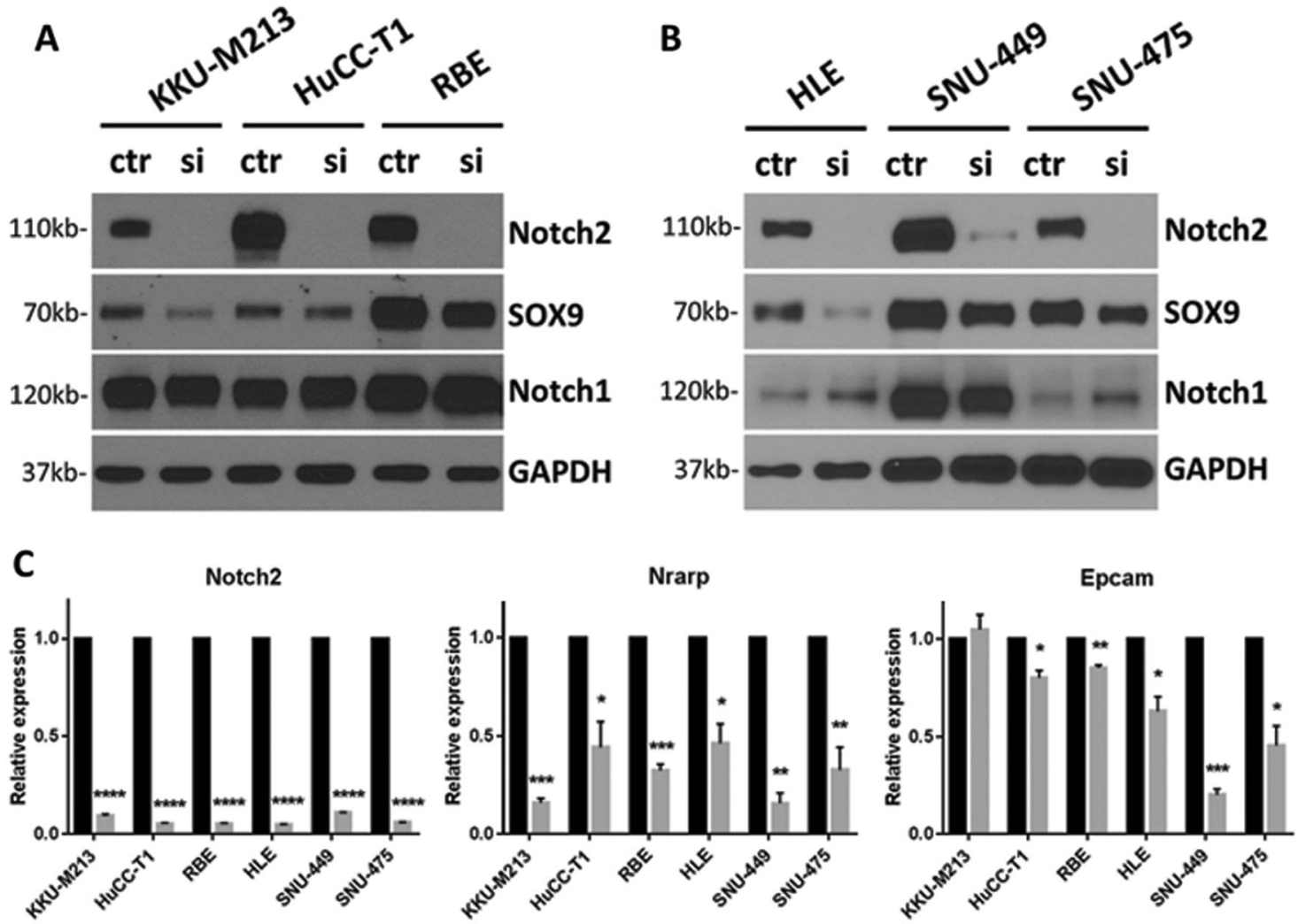

Hes1
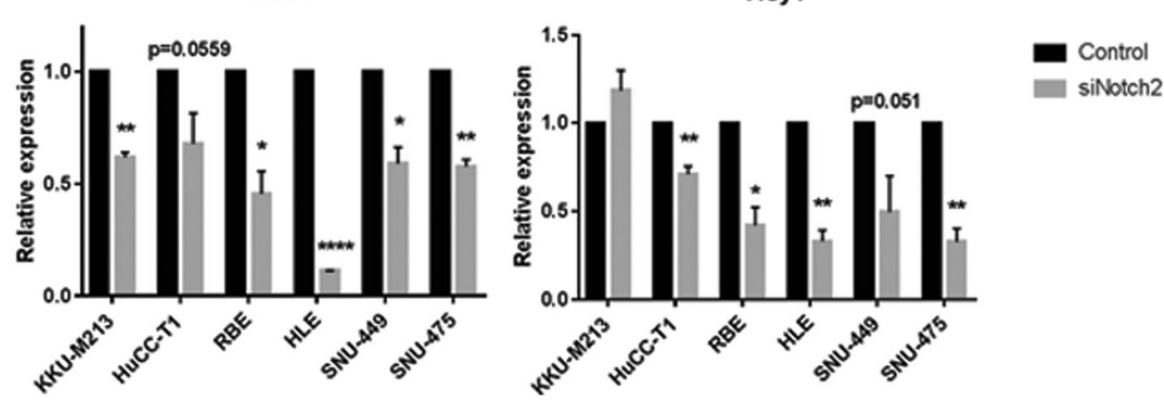

Fig. 6 Inactivation of Notch2 in human intrahepatic cholangiocarcinoma (ICC) and hepatocellular carcinoma (HCC) cell lines. $\mathbf{a}$ and $\mathbf{b}$ Western blot analysis shows the complete inhibition of Notch2 expression in ICC and HCC cell lines transfected with siNotch2. Control is presented as ctr and siNotch 2 group is indicated as si.

derived ICC [6, 7]. However, all these studies were based on gain of function of Notch receptors, aiming to demonstrate that activation of Notch is sufficient to promote ICC formation. Whether Notch is required for hepatocyte-driven ICC development, and if so, which Notch receptor is responsible for this process have not been previously investigated. In this study, by co-expressing AKT and Yap oncogenes with dnRBPJ, which blocks the canonical Notch cascade [28, 29], we convincingly proved that the endogenous Notch signaling is required for hepatocyte-derived ICC. It is worth to note that Notch was found to be required for acinar-to-ductal metaplasia in a KRas-driven PDAC
GAPDH was used as a loading control; $n=3$. $\mathbf{c}$ qPCR analysis of Notch2, Nrarp, Epcam, Hes1, and Hey1. Data are analyzed and normalized using the $-\Delta \Delta \mathrm{Ct}$ method, $n=3$. All data are presented as mean \pm SD. ns $p>0.05 ; * p<0.05 ; * * p<0.01 ; * * * p<0.001 ; * * * * p<$ 0.0001

murine model [36]. In addition, a recent study showed that activation of endogenous Notch leads to transdifferentiation of neuroendocrine to non-neuroendocrine cells in small-cell lung cancer cells [37]. Together these studies point to Notch signaling as an important cell-fate determinant in multiple tumor types.

Furthermore, we investigated which Notch receptor is responsible for Notch-dependent-ICC development, focusing on Notch1 and Notch2 receptors. Using conditional Notch1 and Notch $2 \mathrm{KO}$ mice, we demonstrated that loss of Notch1 only moderately delays AKT/Yap ICC development. However, ICC lesions eventually occurred in all 
mouse livers depleted of Notch1. In striking contrast, when Notch2 was knocked-out in hepatocytes, not only it led to delayed tumor development, but also resulted in the exclusive formation of hepatocellular adenoma and HCClike lesions, thus recapitulating the phenotype observed following the inhibition of the canonical Notch signaling. These observations indicate that the cell autonomous Notch2 is required for hepatocyte-BEC trans-differentiation and ICC formation in mice. In addition, using human HCC and ICC cell lines, we showed that silencing of Notch2 decreased the expression of the BEC marker Sox9. We then analyzed Notch2 expression in relationship to Sox 9 in a human TCGA dataset containing 59 non-tumor livers and 36 human ICC samples [38]. Importantly, a statistically significant correlation between Notch2 and Sox 9 expression in all liver samples, as well as in ICC tumors was found (Sup Figs. 5A and 5B). Furthermore, analysis of the cBioPortal for Cancer Genomics dataset [39] revealed Notch2 amplification in 4/36 (11\%) human ICC specimens, whereas no alterations were identified for Notch1 (Sup Fig. 5C). In humans, loss-of-function mutations in Notch2 or its ligand Jagged1 are associated with Alagille Syndrome, a developmental disorder leading to bile duct scarcity [40, 41]. These data support the prominent role of Notch2 in BEC differentiation and bile duct formation during development, and are consistent with the hypothesis that Notch2 may also control the BEC fate along human cholangiocarcinogenesis. The mechanisms whereby Notch 2 promotes BEC differentiation and ICC development remain to be better delineated. Interestingly, the importance of the JNK signaling in biliary carcinogenesis has been recently demonstrated [30]. Of note, we found that the JNK pathway is impaired in Notch2-depleted livers (Fig. 5f), thus suggesting a functional crosstalk between these two cascades during ICC formation and/or progression. Additional investigation is necessary to unravel the function of the JNK signaling downstream of Notch2 in cholangiocarcinogenesis.

Unlike Notch2, the functional role of Notch1 in intrahepatic cholangiocarcinogenesis remains poorly understood. Using conditional Notchl KO mice, we showed that loss of Notch1 in tumor cells delays AKT/Yap-induced tumor development and mildly reduces the levels of a subset of Notch target genes. These results suggest that the contribution of Notch1 receptor to hepatocyte-derived ICC formation in mice might be limited. Intriguingly, IHC revealed that Notch 1 is predominantly expressed in the tumor microenvironment (Figs. 2c and 4e). This staining pattern in AKT/Yap lesions is consistent with that in AKT/ Ras liver tumors [24]. However, due to the low sensitivity of the anti-Notch1 antibody, we were unable to convincingly conclude whether Notch1 is expressed in ECs or tumor-associated fibroblast cells. Further studies, using single cell RNA-Seq analysis for instance, will be required to identify the cell types expressing Notch1. Furthermore, using proper cell-lineage Cre mouse lines in combination with conditional Notchl KO mice, it would be possible to unravel the precise mechanisms whereby Notch1 plays a role in cholangiocarcinogenesis. For instance, Lrat-Cre mice express Cre in fibroblasts, including hepatic stellate cells [42], and they can be used to delete Notchl in ICCassociated fibroblasts. Also, Tie2-Cre mice [43] could be used to delete Notchl in ECs. In summary, our investigation suggests that Notch1 acts in a paracrine fashion to regulate ICC development.

Finally, our study has important clinical implications. Targeting Notch has been proven highly toxic in tumor patients mainly at the gastrointestinal level to date, presumably due to the important physiological roles of various Notch-related proteins [14]. The present findings suggest that Notch2 might be a potential target in human ICC. Of note, promising anti-neoplastic effects by Notch2-specific antibodies were detected in ICC lesions from AKT/Ras mice [24]. Thus, further studies should be conducted to establish the eventual relevance of anti-Notch2 strategies for the treatment of this deadly disease.

\section{Materials and methods}

\section{Constructs and reagents}

We used constructs as described previously, including pT3EF1 $\alpha, \quad$ pT3-EF1 $\alpha$-HA-myr-AKT (mouse), pT3-EF1 $\alpha-$ YapS127A (human), pT3-EF1 $\alpha$-dnRBPJ (human), pCMV, pCMV-Cre, and pCMV/SB transposase [28, 44-46]. Adeno-associated virus encoding Cre-recombinase under the control of hepatocyte-specific thyroxine-binding globulin ( Tbg) promoter (AAV8-Tbg-Cre) was provided by the University of Pennsylvania Vector Core (Philadelphia, PA). All plasmids were purified utilizing the Endotoxin Free Maxi prep kit (Sigma- Aldrich, St. Louis, MO).

\section{Animals}

We obtained R26R-EYFP mice from Jackson Laboratory (Sacramento, CA, USA). Five female R26R-EYFP mice were injected with AAV8-Tbg-Cre. HA tagged AKT, YapS127 plasmid (AKT/Yap), and SB were delivered to the mice by hydrodynamic injection. FVB/N and Notch $2^{\text {flox/flox }}$ mice were purchased from Jackson Laboratory. Notch flox ${ }^{f l o x}$ mice were a generous gift by Dr. Rong Wang at UCSF. Five female FVB/N mice were injected with AKT/Yap hydrodynamically; 10 male mice were randomly assigned to AKT/Yap/pT3 and AKT/Yap/dnRBPJ groups. A total of 9 female and 9 male Notch2 $2^{\text {floxflox }}$ mice were randomly assigned to AKT/Yap/pCMV and AKT/Yap/Cre groups. 
Finally, 7 female and 13 male Notch $f^{\text {floxfflox }}$ mice were randomly assigned to AKT/Yap/pCMV and AKT/Yap/Cre cohorts. The study was not blinded.

\section{Hydrodynamic tail vein injection}

We employed hydrodynamic injection as previously described in detail [47]. The dosage of pT3-EF1 $\alpha$-HA-myrAKT (AKT) and pT3-EF1 $\alpha$-YapS127A (Yap) plasmids was 20 and $30 \mu \mathrm{g}$, respectively. To suppress the canonical Notch signaling, we applied $60 \mu \mathrm{g}$ pT3-EF1 $\alpha$-dnRBPJ or $60 \mu \mathrm{g}$ pT3-EF1 $\alpha$ together with AKT and Yap plasmids in FVB/N mice. In transgenic models, we injected $60 \mu \mathrm{g}$ pCMV-Cre or $60 \mu \mathrm{g}$ empty pCMV vector together with AKT/Yap. Mice were housed, fed, and monitored according to protocols approved by the Committee for Animal Research at the University of California San Francisco (San Francisco, CA).

\section{Histology, IHC and IF}

Samples were fixed overnight with Zinc Formal-Fixx (Thermo Shandon Limited, Runcorn) at $4{ }^{\circ} \mathrm{C}$ for subsequent paraffin-embedding. Sections were done at $5 \mu \mathrm{m}$ in thickness. For IHC of HNF-4 $\alpha$, antigen unmasking was performed in Tris/EDTA buffer (pH 9.0), while for all other targets in sodium citrate buffer ( $\mathrm{pH}$ 6.0). Other information could be found in the Supplementary Material and Methods.

\section{Protein extraction and western blot analysis}

Specific protein extraction procedures can be found in Supplementary Material and Methods. Aliquots of $30 \mu \mathrm{g}$ lysate were denatured by boiling in Tris-Glycine SDS Sample Buffer (Invitrogen, Grand Island, NY). We used SDS-PAGE for protein separation. And then protein was transferred onto nitrocellulose membranes (Invitrogen). Membranes were blocked in 5\% non-fat dry milk in Trisbuffered saline containing $0.1 \%$ Tween 20 for $1 \mathrm{~h}$ and incubated with specific primary antibodies (Supplementary Table 1). Subsequently, a horseradish peroxidase-secondary antibody diluted 1:10,000 for $1 \mathrm{~h}$ was applied and revealed using the Super Signal West Pico Chemiluminescent Substrate (Pierce Chemical Co., New York, NY).

\section{RNA extraction and qPCR}

We extracted total mRNA from liver tissues and cells using the Quick RNA miniprep kit (Zymo Research, Irvine, CA, USA). Next, mRNA expression was detected by qRT-PCR using SYBR Green Master Mix (Applied Biosystems, Foster City, CA, USA) in an QuantStudio ${ }^{\mathrm{TM}} 6$ Flex system (Applied Biosystems). Expression of each gene was normalized with the $18 \mathrm{~S}$ rRNA. Other conditions can be found in Supplementary Material and Methods. The list of primers is reported in Supplementary Table 2.

\section{Cell culture studies}

We obtained the KKU-M213 and RBE ICC cell lines from the Japanese Collection of Research Bioresources Cell Bank (JCRB, Japan) and RIKEN cell bank (Tsukuba, Japan), respectively. HuCC-T1 was a generous gift by Dr. Gregory J. Gores (Mayo Clinic, Rochester, MN). HLE, SNU-449, and SNU-475 HCC cell lines were purchased from American Type Culture Collection (ATCC, Manassas, VA, USA). All cells were authenticated and tested clear of mycoplasma contamination. Cells were cultured separately in DMEM medium (Gibco, Grand Island, NY, USA) with $5 \%$ fetal bovine serum (Gibco), $100 \mu \mathrm{g} / \mathrm{ml}$ streptomycin, and $100 \mathrm{U} / \mathrm{ml}$ penicillin at $37{ }^{\circ} \mathrm{C}$ in $5 \% \mathrm{CO}_{2}$ humidified incubator.

\section{In vitro siRNA transfection}

KKU-M213, HuCC-T1, RBE ICC cell lines, and HLE, SUN-449, SNU-475 HCC cells were transfected with short interfering RNAs (siRNAs) targeting Notch2 (Thermo Fisher Scientific) or negative control siRNA (Thermo Fisher Scientific) using Lipofectamine RNA/iMAX (Thermo Fisher Scientific). Cells were harvested after $48 \mathrm{~h}$ for qPCR and Western blot analysis.

\section{Statistical analysis}

The Prism 7.0 software (GraphPad, San Diego, CA) was used to analyze the data, which are presented as Means \pm SD. Comparisons between two groups were performed with two-tailed unpaired $t$ test. Welch correction was applied when necessary. $P$ values $<0.05$ were considered statistically significant.

Acknowledgements We would like to thank Dr. Rong Wang at UCSF for providing Notch $1^{f l o x / f l o x}$ mice and Dr. Gregory J. Gores at Mayo Clinic for providing the HuCC-T1 cell line. This study is supported by NIH grants R01CA190606 to X.C., P30DK026743 for UCSF Liver Center; and China Scholarship Council State Scholarship Fund No. 201606550015 to J.W.

\section{Compliance with ethical standards}

Conflict of interest The authors declare that they have no conflict of interest.

Open Access This article is licensed under a Creative Commons Attribution 4.0 International License, which permits use, sharing, adaptation, distribution and reproduction in any medium or format, as long as you give appropriate credit to the original author(s) and the source, provide a link to the Creative Commons license, and indicate if changes were made. The images or other third party material in this 
article are included in the article's Creative Commons license, unless indicated otherwise in a credit line to the material. If material is not included in the article's Creative Commons license and your intended use is not permitted by statutory regulation or exceeds the permitted use, you will need to obtain permission directly from the copyright holder. To view a copy of this license, visit http://creativecommons. org/licenses/by/4.0/.

\section{References}

1. Bridgewater J, Galle PR, Khan SA, Llovet JM, Park JW, Patel T, et al. Guidelines for the diagnosis and management of intrahepatic cholangiocarcinoma. J Hepatol. 2014;60:1268-89.

2. Sia D, Villanueva A, Friedman SL, Llovet JM. Liver cancer cell of origin, molecular class, and effects on patient prognosis. Gastroenterology. 2017;152:745-61.

3. Chen Y, Wong PP, Sjeklocha L, Steer CJ, Sahin MB. Mature hepatocytes exhibit unexpected plasticity by direct dedifferentiation into liver progenitor cells in culture. Hepatology. 2012;55:563-74.

4. Tarlow BD, Pelz C, Naugler WE, Wakefield L, Wilson EM, Finegold MJ, et al. Bipotential adult liver progenitors are derived from chronically injured mature hepatocytes. Cell Stem Cell. 2014;15:605-18.

5. Palmer WC, Patel T. Are common factors involved in the pathogenesis of primary liver cancers? A meta-analysis of risk factors for intrahepatic cholangiocarcinoma. J Hepatol. 2012;57:69-76.

6. Fan B, Malato Y, Calvisi DF, Naqvi S, Razumilava N, Ribback S, et al. Cholangiocarcinomas can originate from hepatocytes in mice. J Clin Invest. 2012;122:2911-5.

7. Sekiya S, Suzuki A. Intrahepatic cholangiocarcinoma can arise from Notch-mediated conversion of hepatocytes. J Clin Invest. 2012;122:3914-8.

8. Zong Y, Panikkar A, Xu J, Antoniou A, Raynaud P, Lemaigre F, et al. Notch signaling controls liver development by regulating biliary differentiation. Development. 2009;136:1727-39.

9. Bray SJ. Notch signalling in context. Nat Rev Mol Cell Biol. 2016;17:722-35.

10. Gil-Garcia B, Baladron V. The complex role of NOTCH receptors and their ligands in the development of hepatoblastoma, cholangiocarcinoma and hepatocellular carcinoma. Biol Cell. 2016;108:29-40.

11. D'Souza B, Miyamoto A, Weinmaster G. The many facets of Notch ligands. Oncogene. 2008;27:5148-67.

12. D'Souza B, Meloty-Kapella L, Weinmaster G. Canonical and non-canonical Notch ligands. Curr Top Dev Biol. 2010;92:73-129.

13. Katsube K, Sakamoto K. Notch in vertebrates-molecular aspects of the signal. Int J Dev Biol. 2005;49:369-74.

14. Cigliano A, Wang J, Chen X, Calvisi DF. Role of the Notch signaling in cholangiocarcinoma. Expert Opin Ther Targets. 2017;21:471-83.

15. Lamar E, Deblandre G, Wettstein D, Gawantka V, Pollet N, Niehrs C, et al. Nrarp is a novel intracellular component of the Notch signaling pathway. Genes Dev. 2001;15:1885-99.

16. Qi R, An H, Yu Y, Zhang M, Liu S, Xu H, et al. Notch1 signaling inhibits growth of human hepatocellular carcinoma through induction of cell cycle arrest and apoptosis. Cancer Res. 2003;63:8323-9.

17. Wang C, Qi R, Li N, Wang Z, An H, Zhang Q, et al. Notch1 signaling sensitizes tumor necrosis factor-related apoptosis-inducing ligand-induced apoptosis in human hepatocellular carcinoma cells by inhibiting Akt/Hdm2-mediated p53 degradation and up-regulating p53-dependent DR5 expression. J Biol Chem. 2009;284:16183-90.

18. Wu WR, Zhang R, Shi XD, Zhu MS, Xu LB, Zeng H, et al. Notch1 is overexpressed in human intrahepatic cholangiocarcinoma and is associated with its proliferation, invasiveness and sensitivity to 5-fluorouracil in vitro. Oncol Rep. 2014;31:2515-24.

19. Croquelois A, Blindenbacher A, Terracciano L, Wang X, Langer I, Radtke F, et al. Inducible inactivation of Notch1 causes nodular regenerative hyperplasia in mice. Hepatology. 2005;41:487-96.

20. Geisler F, Nagl F, Mazur PK, Lee M, Zimber-Strobl U, Strobl LJ, et al. Liver-specific inactivation of Notch2, but not Notch1, compromises intrahepatic bile duct development in mice. Hepatology. 2008;48:607-16.

21. Kodama Y, Hijikata M, Kageyama R, Shimotohno K, Chiba T. The role of notch signaling in the development of intrahepatic bile ducts. Gastroenterology. 2004;127:1775-86.

22. Jeliazkova P, Jors S, Lee M, Zimber-Strobl U, Ferrer J, Schmid RM, et al. Canonical Notch2 signaling determines biliary cell fates of embryonic hepatoblasts and adult hepatocytes independent of Hes1. Hepatology. 2013;57:2469-79.

23. Wu WR, Shi XD, Zhang R, Zhu MS, Xu LB, Yu XH, et al. Clinicopathological significance of aberrant Notch receptors in intrahepatic cholangiocarcinoma. Int $J$ Clin Exp Pathol. 2014;7:3272-9.

24. Huntzicker EG, Hotzel K, Choy L, Che L, Ross J, Pau G, et al. Differential effects of targeting Notch receptors in a mouse model of liver cancer. Hepatology. 2015;61:942-52.

25. Zhang S, Song X, Cao D, Xu Z, Fan B, Che L, et al.Pan-mTOR inhibitor MLN0128 is effective against intrahepatic cholangiocarcinoma induced in mice. J Hepatol. 2017;67:1194-1203.

26. Malato Y, Naqvi S, Schurmann N, Ng R, Wang B, Zape J, et al. Fate tracing of mature hepatocytes in mouse liver homeostasis and regeneration. J Clin Invest. 2011;121:4850-60.

27. Zender S, Nickeleit I, Wuestefeld T, Sorensen I, Dauch D, Bozko $\mathrm{P}$, et al. A critical role for notch signaling in the formation of cholangiocellular carcinomas. Cancer Cell. 2013;23:784-95.

28. Che L, Fan B, Pilo MG, Xu Z, Liu Y, Cigliano A, et al. Jagged 1 is a major Notch ligand along cholangiocarcinoma development in mice and humans. Oncogenesis. 2016;5:e274.

29. Morell CM, Fiorotto R, Fabris L, Strazzabosco M. Notch signalling beyond liver development: emerging concepts in liver repair and oncogenesis. Clin Res Hepatol Gastroenterol. 2013;37:447-54.

30. Yuan D, Huang S, Berger E, Liu L, Gross N, Heinzmann F, et al. Kupffer cell-derived Tnf triggers cholangiocellular tumorigenesis through JNK due to chronic mitochondrial dysfunction and ROS. Cancer Cell. 2017;31:771-89. e776

31. Michalopoulos GK, Barua L, Bowen WC. Transdifferentiation of rat hepatocytes into biliary cells after bile duct ligation and toxic biliary injury. Hepatology. 2005;41:535-44.

32. Yanger K, Zong Y, Maggs LR, Shapira SN, Maddipati R, Aiello NM, et al. Robust cellular reprogramming occurs spontaneously during liver regeneration. Genes Dev. 2013;27:719-24.

33. Merrell AJ, Stanger BZ. Adult cell plasticity in vivo: dedifferentiation and transdifferentiation are back in style. Nat Rev Mol Cell Biol. 2016;17:413-25.

34. Storz P. Acinar cell plasticity and development of pancreatic ductal adenocarcinoma. Nat Rev Gastroenterol Hepatol. 2017;14:296-304.

35. Wong $\mathrm{CH}, \mathrm{Li} \mathrm{YJ}$, Chen YC. Therapeutic potential of targeting acinar cell reprogramming in pancreatic cancer. World J Gastroenterol. 2016;22:7046-57.

36. Avila JL, Troutman S, Durham A, Kissil JL. Notch1 is not required for acinar-to-ductal metaplasia in a model of Krasinduced pancreatic ductal adenocarcinoma. PLoS One. 2012;7: e52133. 
37. Lim JS, Ibaseta A, Fischer MM, Cancilla B, O’Young G, Cristea $S$, et al. Intratumoural heterogeneity generated by Notch signalling promotes small-cell lung cancer. Nature. 2017;545:360-4.

38. Farshidfar F, Zheng S, Gingras MC, Newton Y, Shih J, Robertson $\mathrm{AG}$, et al. Integrative genomic analysis of cholangiocarcinoma identifies distinct IDH-mutant molecular profiles. Cell Rep. 2017;19:2878-80.

39. Cerami E, Gao J, Dogrusoz U, Gross BE, Sumer SO, Aksoy BA, et al. The cBio cancer genomics portal: an open platform for exploring multidimensional cancer genomics data. Cancer Discov. 2012;2:401-4.

40. Li L, Krantz ID, Deng Y, Genin A, Banta AB, Collins CC, et al. Alagille syndrome is caused by mutations in human Jagged1, which encodes a ligand for Notch1. Nat Genet. 1997;16:243-51.

41. McDaniell R, Warthen DM, Sanchez-Lara PA, Pai A, Krantz ID, Piccoli DA, et al. NOTCH2 mutations cause Alagille syndrome, a heterogeneous disorder of the notch signaling pathway. Am J Hum Genet. 2006;79:169-73.

42. Mederacke I, Hsu CC, Troeger JS, Huebener P, Mu X, Dapito $\mathrm{DH}$, et al. Fate tracing reveals hepatic stellate cells as dominant contributors to liver fibrosis independent of its aetiology. Nat Commun. 2013;4:2823.

43. Kisanuki YY, Hammer RE, Miyazaki J, Williams SC, Richardson JA, Yanagisawa M. Tie2-Cre transgenic mice: a new model for endothelial cell-lineage analysis in vivo. Dev Biol. 2001;230:230-42.

44. Hu J, Che L, Li L, Pilo MG, Cigliano A, Ribback S, et al. Coactivation of AKT and c-Met triggers rapid hepatocellular carcinoma development via the mTORC1/FASN pathway in mice. Sci Rep. 2016;6:20484.

45. Marti P, Stein C, Blumer T, Abraham Y, Dill MT, Pikiolek M, et al. YAP promotes proliferation, chemoresistance, and angiogenesis in human cholangiocarcinoma through TEAD transcription factors. Hepatology. 2015;62:1497-510.

46. Tao J, Calvisi DF, Ranganathan S, Cigliano A, Zhou L, Singh S, et al. Activation of beta-catenin and Yap1 in human hepatoblastoma and induction of hepatocarcinogenesis in mice. Gastroenterology. 2014;147:690-701.

47. Chen X, Calvisi DF. Hydrodynamic transfection for generation of novel mouse models for liver cancer research. Am J Pathol. 2014;184:912-23. 